\title{
Gradients in the expression of cell surface glycoproteins in a simple tissue, the Dictyostelium discoideum slug
}

\author{
Lois H. Browne and KeITH L. Williams* \\ School of Biological Sciences, Macquarie University, Sydney 2109, NSW, Australia \\ (Received 9 October 1992; revised 18 December 1992; accepted 6 January 1993)
}

\begin{abstract}
Gradients in diffusible morphogens have long been postulated to have a role in the establishment of polarity and pattern formation in developing animal embryos. In the cellular slime mould Dictyostelium discoideum, several morphogens have been described and their interactions and location in the multicellular 'slug' stage are being studied. Here we report that two cell surface antigens, which identify prespore and prestalk cells, respectively, are present at different levels on the surface of cells depending on their position within the slug. This is the first evidence of the existence of gradients in cell surface molecules in Dictyostelium discoideum tissues. The level of prespore glycoprotein PsA on the surface of prespore cells increased progressively along the length of the slug, so that the rear cells had the highest surface density of this glycoprotein. Since the pspA gene, which encodes PsA, is cAMPregulated, this raises the possibility that expression of PsA reflects an underlying morphogenetic cAMP gradient. The level of the MUD9 antigen, which is prestalk-enriched, decreased on the surface of prestalk cells towards the rear of the slug. As this correlated with a decrease in size of these cells along the length of the slug, it is possible that the surface density of this antigen is approximately constant. We have also identified a population of prestalklike cells that lack both the PsA glycoprotein and the MUD9 antigen. These cells primarily occurred in the posterior of the slug and could represent the population of cells that form the basal disc.
\end{abstract}

\section{Introduction}

The cellular slime mould Dictyostelium discoideum consists of single-celled amoebae during the feeding stage of its life cycle; as food becomes scarce the cells aggregate together to form a multicellular 'slug' consisting of about $10^{5}$ cells (Raper, 1984). These slugs are motile, but eventually form a sessile fruiting body. Two principal cell types exist within the slug, the prespores and prestalk cells which will form the spore head and the stalk respectively of the fruiting body. Recently the prestalk cells at the anterior of the slug have been shown to be heterogeneous on the basis of expression of genes encoding secreted proteins ST310 and ST430 (Jermyn et al., 1989).

Studies on the establishment of spatial cell patterning in $D$. discoideum have in the past centred on diffusible morphogens including cAMP (Kay et al., 1978) and DIF (Morris et al., 1987) and to a lesser extent adenosine (Schaap \& Wang, 1986) and $\mathrm{NH}_{3}$ (Gross et al., 1983). By contrast, the emphasis in animal systems has been on the

\footnotetext{
*Author for correspondence. Tel. (02) 805 8212; fax (02) 8058174 ;
} email williams@angis.su.oz.au. role of gene products in tissue organization (NussleinVolhard, 1991). Retinoic acid has been considered to be the first vertebrate morphogen (Thaller \& Eichele, 1987), but recent evidence raises some controversy on this issue (Wanek et al., 1991; Noji et al., 1991).

In $D$. discoideum two cell surface glycoproteins have been found using monoclonal antibodies (mAbs) to identify prestalk (Krefft et al., 1985) and prespore cells (Krefft et al., 1984). One of these, the prespore surface glycoprotein PsA, is well characterized (Gooley et al., 1992). It is likely that the expression of these cell surface antigens is important in tissue formation, and so their spatial expression within the slug is examined here.

We found non-uniform expression in the cell surface distribution of both of these molecules along the long axis of the slug. In addition, a sub-population of cells which lack either antigen was discovered at the rear of the slug.

\section{Methods}

$m A b s$. mAbs MUDI, which recognizes a glycoprotein prespore antigen (PsA) found only in prespore cells (Krefft et al., 1984), and MUD9, which binds to a glycoprotein not as yet characterized but known to be present at higher levels on prestalk cells than prespore cells 

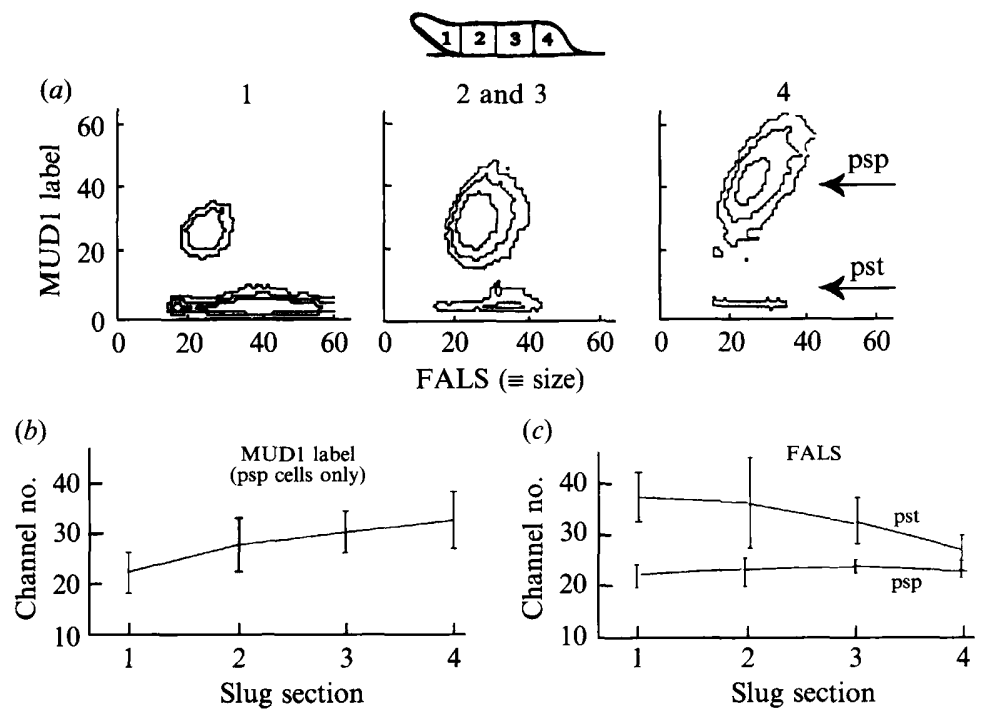

Fig. 1. (a) Dual parameter flow cytometry histograms of cells from slug regions 1,2 and 3 combined, and 4 . The $x$-axis represents FALS (correlated with cell size), the y-axis mAb labelling with MUD1-FITC (PsA) on the surface of the cells. For both axes, the channel numbers are arbitrary units. Total cell count was about 40000 and the three contour lines represent cell counts above 15,25 and 50, respectively. The cells which label with MUD1 (PsA) are the prespore cells (psp). The mean level of PsA on the cell surface of these cells increases towards the posterior of the slug (see $b$ ), while the FALS of these cells remain similar $(c)$. The cells not labelled by MUD1, called here for convenience the prestalk cells (pst), are more abundant in the anterior. There is a decrease in mean FALS of these cells towards the posterior of the slug $(c)$. (b) Flow-cytometer-generated mean MUD1 labelling on the cell surface of the prespore cells for slug regions 1-4. Error bars represent \pm 2 SEM for 3-5 estimates of each slug region. $(c)$ Flow-cytometer-generated mean FALS of the prespore and prestalk cells for slug regions 1-4. Error bars represent \pm 2 SEM for 3-5 estimates of each slug region.

(Krefft et al., 1985), were prepared and conjugated with fluorescein isothiocyanate (FITC) or $R$-phycoerythrin (PE) as previously described (Bernstein et al., 1988).

Flow analysis of cells. Migrating D. discoideum slugs of strain WS380B each comprising about $10^{5}$ cells were prepared as previously described (Browne et al., 1989). Slugs were divided into four sections of approximately equal length from anterior to posterior using a flattened platinum wire. About four slugs were matched for size and age (determined by distance of migration from the origin) and combined for flow cytometry. 'Young' slugs were those which had completely left the origin but had migrated less than $2 \mathrm{~cm}$ (less than $48 \mathrm{~h}$ ) and were elongated in shape (Breen et al., 1992). Mature slugs had migrated between 2 and $4 \mathrm{~cm}$ (approx. 2-4 d) from the origin.

Slug samples were disaggregated to single cells and labelled as previously described (Browne et al., 1989) with either FITC-coupled MUD1 or FITC-coupled MUD9. For two-colour analysis, FITCcoupled MUD1 and PE-coupled MUD9 were used. Cells were analysed without further washing using a Coulter Epics V flow cytometer. For single-colour analysis each cell was analysed for two variables: forward angle light scatter (FALS) as a measure of relative size, and integrated green fluorescence as a measure of number of $\mathrm{mAb}$ molecules attached. Two-colour analysis was as described in Bernstein et al. (1988).

\section{Results}

If the expression of cell surface glycoproteins reflects spatial morphogen gradients, it might be expected that these molecules will not be uniformly distributed along the slug. This prediction was borne out for PsA on prespore cells and the MUD9 antigen on prestalk cells. This was not the case for the MUD9 antigen on prespore cells as it was expressed at a constant low level along the slug.

\section{PsA levels on the surface of prespore cells show a gradient along the slug}

Flow cytometry displays of FALS, a measure of size, vs mAb MUD1 label (which specifically identifies glycoprotein PsA) showed that the average number of molecules of PsA on the surface of prespore cells increased from the anterior to the posterior of the slug (Fig. 1a). Data from at least three experiments showing the mean levels of MUD1 label along the slug confirmed this conclusion (Fig. 1b). A trivial explanation for this change could be that prespore cells are different in size along the slug. This was shown not to be so, as there was no significant change in peak FALS (an indication of size) of the prespore cells in different sections (Fig. 1c). Therefore, the change in levels of PsA represents a change in density of surface expression.

There was a clear discontinuity between prespore cells and other cells (commonly called prestalk cells, but see 

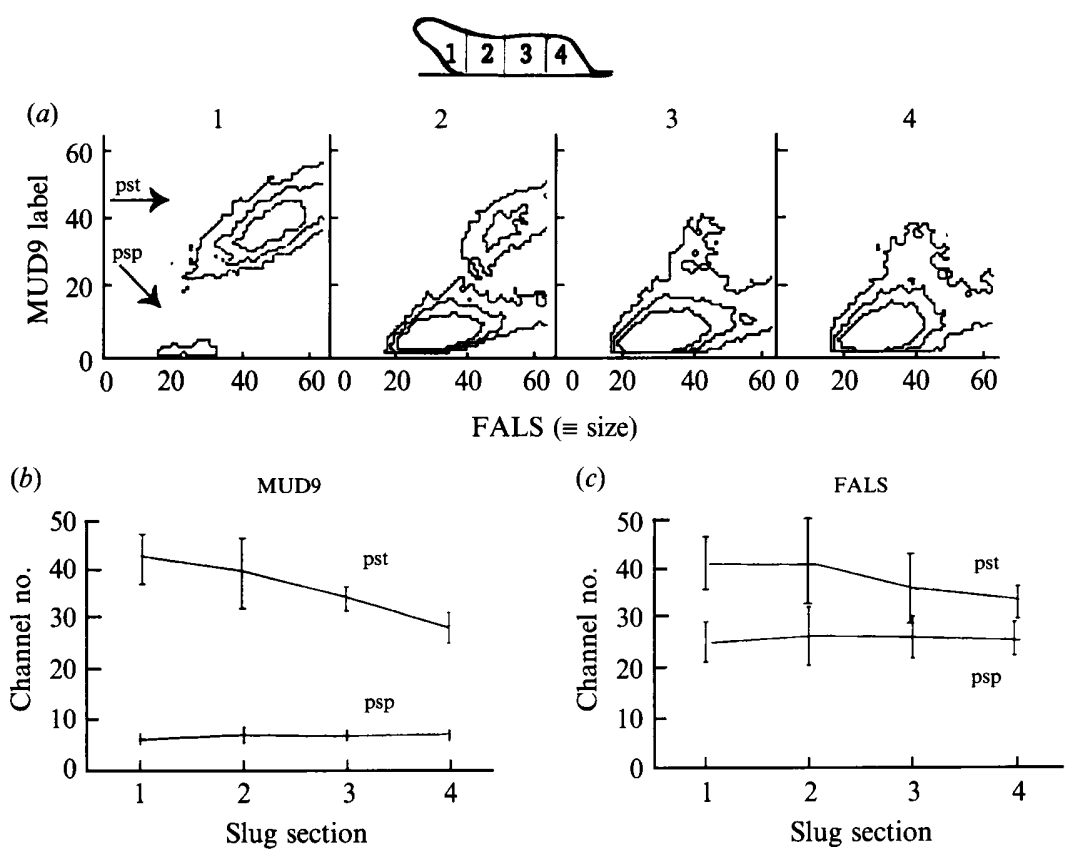

Fig. 2. (a) Dual parameter flow cytometry histograms of cells from slug regions 1-4. The x-axis represents FALS (correlated with cell size) and the $y$-axis mAb labelling with MUD9-FITC on the surface of the cells. The channel numbers are arbitrary units. For each plot, approximately 20000 cells were collected, and the three contour lines represent cell counts above 5,10 and 20 , respectively. MUD9-labelled cells are the prestalk cells (pst) (see text). The percentage of these cells, the mean level of the MUD9 antigen on the cell surface of these cells $(b)$ and the FALS $(c)$ all decrease towards the posterior of the slug. Most of the cells with low levels of MUD9 label are prespore cells (psp), which label with MUD1 (see Fig. 1). (b) Flow-cytometer-generated mean MUD9 labelling on the cell surface of prestalk cells and prespore cells for slug regions 1-4. The error bars represent \pm 2 SEM for 3-5 estimates of each slug region. (c) Mean FALS of prespore and prestalk cells for slug regions 1-4. The error bars represent \pm 2 SEM for 3-5 estimates of each slug region. NB Prestalk cells, although clearly present in regions 3 and 4, did not form a discrete sub-population, and therefore the mean channel numbers for these cells were calculated with less accuracy than those for regions 1 and 2 .

later) based on MUD1 staining. The prestalk cells in all sections were unlabelled with MUD1.

\section{MUD9 antigen on the prestalk cell surface shows a gradient along the slug}

Flow cytometry displays of FALS vs MUD9 label showed that the level of MUD9 antigen on the surface of prestalk cells was highest at the anterior and decreased towards the posterior of the slug (Fig. 2a). Data from at least three experiments are summarized in Fig. $2(b)$ in which mean peak MUD9 levels along the slug are plotted. The average size (FALS) of prestalk cells decreased towards the posterior (Fig. $2 a, c$ ). The same decrease in size of prestalk cells along the slug is apparent in Fig. $1(a, c)$ where cells were labelled with MUD1 (prestalk cells are those lacking MUD1). As both the size of cells and the levels of MUD9 expression decreased towards the posterior it may be that the density of the MUD9 antigen on the cell surface remains constant. Hence, unlike the situation observed for PsA on the prespore surface, the gradient of MUD9 label on prestalk cells may reflect a change in size of these cells along the slug.
Fig. 2(b) also displays the mean levels of MUD9 label on the surface of the prespore cells, which while present at low levels showed no significant difference along the slug length. It is important to note that prespore cells do not change in size along the slug (Figs $1 c$ and $2 c$ ).

\section{Surface antigen levels vary with slug age}

PsA levels on the surface of prespore cells increased as the slugs matured (Fig. 3). The prespore cells of elongated slugs just out of the pick, i.e. slugs which had not yet drawn up their tail to become compact, had very low levels of PsA on the cell surface. In fact, the level of PsA in young slugs was found to be low in all sections and no significant difference could be detected between anterior and posterior zones in terms of MUD1 staining (data not shown). Mature slugs showed the gradient of cell surface molecules along the slug length as described in Figs 1 and 2.

The levels of the MUD9 antigen on the surface of prestalk cells did not vary significantly between young elongated slugs and mature slugs (data not shown). 
(a)

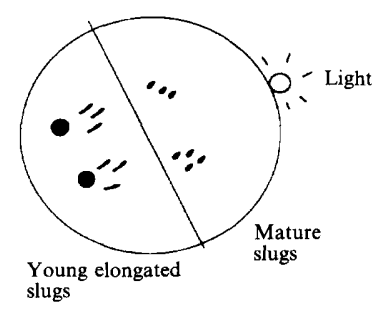

(b)

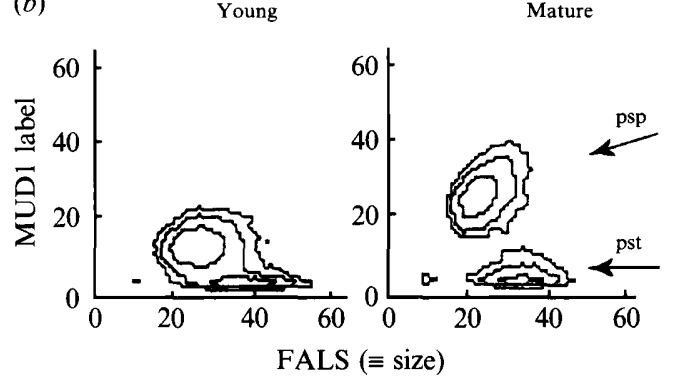

(c)

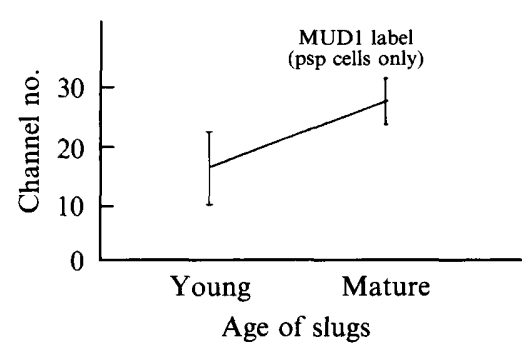

Fig. 3 (a) Schematic diagram of migrating slugs on a water agar plate. The large circle represents a $9 \mathrm{~cm}$ Petri dish, while the black spots are regions where Klebsiella aerogenes and amoebae of $D$. discoideum were deposited on the agar to aggregate, form slugs and migrate towards the lateral light source. (b) Dual parameter flow cytometry histograms of cells from young and mature slugs. The $\mathrm{x}$-axis represents FALS (correlated with cell size), the y-axis labelling with MUD1-FITC (PsA) on the surface of the cells. The channel numbers are arbitrary units. For each plot about 25000 cells were collected and the three contour lines represent cell counts above 10,20 and 50 , respectively. The cells which label with MUD1 (PsA) are the prespore cells (psp). The mean level of PsA on the cell surface of prespore cells of young, elongated slugs is less than that of mature slugs. The cells not labelled by MUD1 are the prestalk cells (pst). (c) Mean MUD1 labelling on the cell surface for young and mature slugs. The error bars represent $\pm 2 \operatorname{SEM}(n=3)$.

\section{Identification of a class of cells lacking both PSA and MUD9 antigen}

Two-colour results (MUD1 and MUD9 labelling) of whole slugs showed that some particles had low levels of both PsA and MUD9 antigen (Fig. 4a, whole slug, section at origin). On analysis of the FALS of these readings, it was apparent that some particles had FALS measurements similar to cells (Fig. $4 b$, whole slug). However, it was difficult to interpret whether this was a valid cell subpopulation as these readings formed a tail of the distribution of the vast majority of measures which peaked in the FALS range indicating cell debris, etc. (Fig. $4 b$ whole slug, inset) and were a small proportion (approx. $5 \%$ ) of the total readings with FALS above threshold levels.

Anterior slug sections had a similar distribution of FALS data for particles lacking MUD1 and MUD9 antigens to that seen in whole slugs (Fig. $4 b, 1$ and 2) where only a small number of particles had sufficient FALS to be cells, and no population curve could be clearly seen. However, sections towards the posterior of the slug (Fig. 4b, 3 and 4) showed that particles lacking both PsA and MUD9 antigen were more abundant (approx. 15\%) and their FALS values formed a distinct distribution curve quite separate and identifiable from those of cell debris (see also Table 1).

The FALS distribution of this subpopulation was similar to that of other cells in these sections. Since both cell types in the rear of the slug had a similar FALS (Figs 1 and 2), it was not possible to identify whether these cells were of prespore (losing PsA) or prestalk (losing MUD9 antigen) origin.

It is concluded that in the posterior half of the slug there is a class of cells lacking the normal cell surface levels of both PsA and MUD9 antigen. The data do not exclude the possibility that a small number of similar cells may also be present in the anterior regions. Prestalk cells (MUD9-positive) had a greater range of both size and MUD9 expression at the anterior (Fig. 2a). Although a discrete cell subpopulation could not be detected, this may reflect heterogeneity of cell type, as the distribution had sufficient spread to include measures similar to those of cells in the posterior sections. Fig. 4(a) ( 3 and 4) and Table 1 also show that the rear of the slug has very few 'classical' prestalk cells, i.e. those with high levels of MUD9 antigen and lacking PsA.

\section{Discussion}

Prespore and prestalk cells have long been known to have a different spatial distribution in the $D$. discoideum slug. This was first established by the use of vital dyes (Bonner, 1952), but more specific markers were found with the advent of the first polyclonal (Takeuchi, 1963) and later monoclonal antibodies and lectins (West \& McMahon, 1979; Gregg et al., 1982; Tasaka et al., 1983). The original view of a simple prestalk anterior and a prespore posterior pattern was superceded when it was realized that there are as many prestalk-like cells in the rear of the slug as there are prestalk cells in the front (Sternfeld \& David, 1982; Voet et al., 1985). Our observations are in accord with those of Takeuchi et al. (1977) that the prestalk cells in the rear lie along the length of the ventral surface. Anterior-like cells at the rear of the slug have been considered to be similar to the 

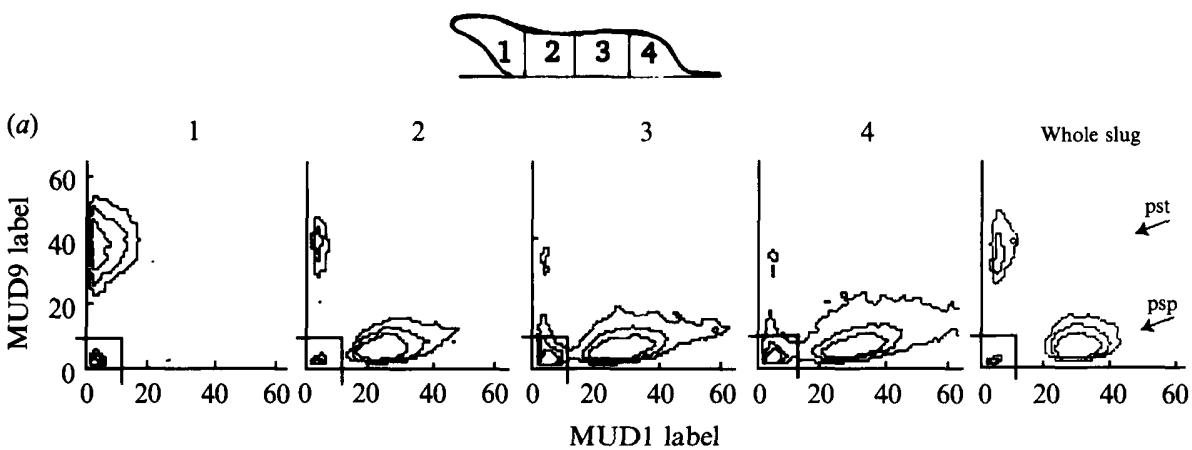

(b)

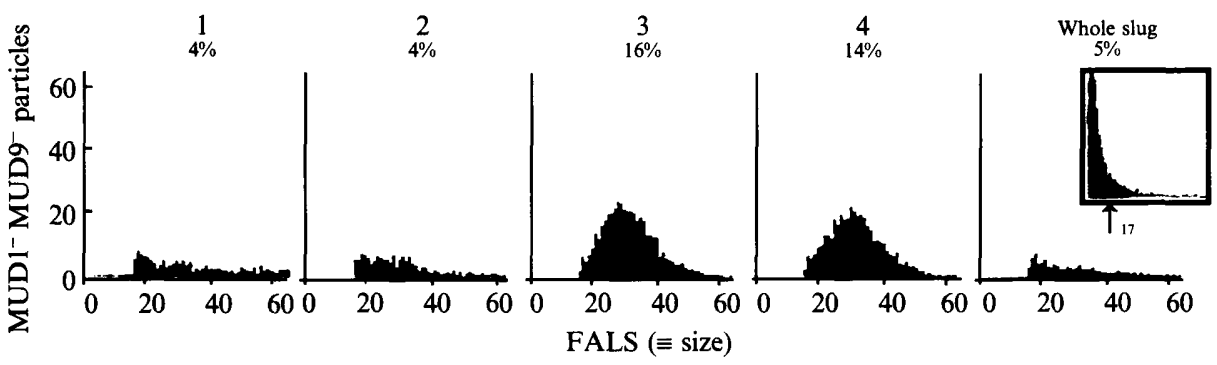

Fig. 4 (a) Dual parameter flow cytometry histograms of cells from slug regions $1-4$ and whole slugs. The axes represent $\mathrm{mAb}$ labelling on the surface of the cells: $x$-axis, MUD1-PE (PsA); y-axis, MUD9-FITC. The channel numbers are arbitrary units. For each plot about 20000 cells were collected and the three contour lines represent cell counts above 5, 20 and 40, respectively. Note that in the example of region 1 illustrated, there were insufficient prespore cells to generate a contour map. The cells which label strongly with MUD1 (PsA) and a little with MUD9 are the prespore cells (psp), and the cells which label with MUD9 and not MUD1 are designated as prestalk cells (pst) (see text). Note that there is a population labelled with neither mAb; FALS for these are shown in $(b)$. (b) Single parameter flow cytometry histograms showing the FALS of the particles ( $a$, boxed) which lack both MUD9 and MUD1 labelling. The profiles shown are for particles with a size above channel number 17, which represents an abitrary cut-off for small cells. Particles with FALS below about channel number 17 are present in all samples and represent very small particles believed to be cell debris, etc. These are not normally displayed in flow cytometry results. The inset for the whole slug sample demonstrates a typical profile below this channel number. The percentages refer to the number of particles with negative MUD1 and MUD9 labelling as a proportion of total particles (cells) which have a FALS above channel number 17. Regions 1 and 2 and whole slugs have a small number of such particles which may represent cells without either label, but the FALS distribution is spread and does not display a definite distribution curve as a sub-population of cells usually does. Regions 3 and 4 in the rear of the slug have a greatly increased number of these particles and display a definite FALS distribution curve which is similar to prespore and prestalk cells in these sections.

Table 1. Percentage of different cell types in the slug

Data represent average percentages from three dual labelling flow cytometry experiments.

\begin{tabular}{llccc}
\hline \hline & $\begin{array}{c}\text { Prespore } \\
\text { cells }\end{array}$ & $\begin{array}{c}\text { Prestalk } \\
\text { cells }\end{array}$ & $\begin{array}{c}\text { New class } \\
\text { of cells }\end{array}$ \\
\hline & Region & $\begin{array}{c}\text { MUD1 }^{+} \\
\text {MUD9 }^{-}\end{array}$ & $\begin{array}{c}\text { MUD1 }^{-} \\
\text {MUD9 }^{+}\end{array}$ & $\begin{array}{c}\text { MUD1 }^{-} \\
\text {MUD9- }^{-}\end{array}$ \\
\hline Whole slug & $1-4$ & 75 & 20 & 5 \\
Slug regions & 1 & 18 & 78 & 4 \\
& 2 and 3 & 78 & 12 & 10 \\
& 4 & 81 & 5 & 14 \\
\hline \hline
\end{tabular}

prestalk cells at the anterior of the slug, although some differences have been suggested in cAMP responsiveness (Sternfeld \& David, 1981) and expression of acid phosphatase (Weijer \& Durston, 1985). In addition to the spatial patterns described above, the prestalk population of the slug is being shown to be increasingly heterogeneous at the molecular level. Jermyn et al. (1989) found three classes of prestalk and anterior-like cells on the basis of expression of two genes encoding extracellular matrix proteins ST310 and ST430; Gomer et al., (1986) found that cells which do not express prestalk cathepsin were located towards the posterior of the anterior 'prestalk' region, and cells which are cAMP independent with respect to stalk formation have been found at the very tip (Kwong et al., 1988).

In the above studies, subpopulations of prestalk cells were found to be qualitatively different, based on different molecules. Whilst two proteins secreted by the prestalk cells, ST310 and ST430, may exist as a gradient in the matrix of the slug, these proteins are entirely extracellular (Early et al., 1988). Here we have presented the first evidence of a cell surface spatial molecular heterogeneity. The expression of the MUD9 antigen on the prestalk cells decreases rearwards. The cells in the rear appeared either to have lost or to be losing the 
MUD9 antigen from the cell surface. It is possible that the prestalk cells are largely responsible for slug movement by fountain flow (Odell \& Bonner, 1986), and that the gradient of MUD9 antigen reflects the different movement profile along the slug.

Density gradient methods for separating disaggregated slug cells often result in more than one band for a cell type. The molecular basis for the difference in density is still unknown. Lam et al. (1981) found slug cells separated into three bands, two of which were identifiable as prespore and prestalk cells, respectively. The third band, which represented $3 \%$ of the cells, was found to be prestalk-like from enzyme assays, and could conceivably be the same class as the cells lacking MUD1 and MUD9 described in this report. From their position in the slug these cells could be precursor cells for the basal disc.

There is no evidence for heterogeneity of prespore cells based on different molecules, but with this report, there are now two reports of heterogeneity in quantitative levels of prespore gene products. The possibility of a gradient of expression of an internal prespore protein, SP60, has been suggested by Haberstroh \& Firtel (1990). This result should be treated with caution, as no gradient was detected with an intact gene present, although studies with mutants containing successive deletions of a repeated CA-rich element and surrounding sequence resulted in a gradient of decreased expression of SP60 from anterior to posterior. This gradient was opposite in direction to that found by us. PsA increases in density on the surface of the prespore cells towards the posterior of the slug. The gradient appears to be significant as the other characteristics of the prespore cells of mature slugs examined (i.e. size and MUD9 expression) were not shown to be spatially regulated.

The challenge now is to see if gradients in cell surface glycoprotein expression (gene activity) reflect morphogenetic gradients. There is a great deal of evidence that cAMP is involved in the induction of gene expression of internal proteins in $D$. discoideum slug cells (reviewed by Williams, 1988) and the cell surface protein PsA is known to be cAMP-regulated (Barklis \& Lodish, 1983). While the anterior of the slug is believed to be the centre of emission of cAMP pulses (Schaap, 1986; Otte et al., 1986), this same region also has greater activity of phosphodiesterase which degrades cAMP (Otte et al., 1986). Although obtaining accurate measurements of extracellular concentrations of cAMP in the slug is difficult, these factors are proposed to result in pulsatile cAMP levels in the anterior of slugs and higher, constant levels of cAMP in the posterior (Wang et al., 1988). Prespore gene induction is believed to require continuous high levels of cAMP (Firtel, 1991). These results raise the possibility of a connection between an extracellular cAMP gradient within the slug and a gradient in the expression of the cell surface glycoprotein PsA. Our results also show that the establishment of the PsA gradient is developmentally regulated, as very young slugs have a lower level of PsA on the cell surface and no gradient was detectable. All of these results are consistent with theoretical predictions that the time taken to establish a Gierer-Meinhardt-type gradient in a slug, which may be $1-2 \mathrm{~mm}$ long, will require many hours (Morrissey, 1982).

Morphogenetic gradients are well described in $D$. discoideum, but no morphogens have yet been definitely established in other systems. In Drosophila, the products of the genes bicoid and dorsal form concentration gradients along the embryo axis, but this is at the syncytial stage of development, and the gradients may arise from diffusion within the cytoplasm (St Johnston \& Nusslein-Volhard, 1992). Very recent evidence for cell surface gradients of adhesive molecules has been reported upon cellularization in insect development (McAllister \& Goodman, 1992). In animal development, which is fundamentally cellular, retinoic acid exists as a shallow gradient in the vertebrate limb bud (Gurdon, 1992) but controversy remains as to whether it is a true morphogen associated with the regulation of gene expression (Wanek et al., 1991; Noji et al., 1991). This work begins the connection between morphogens and cell surface molecules, in this case the PsA glycoprotein of prespore cells and the MUD9 antigen of prestalk cells of $D$. discoideum.

PsA is a major cell surface molecule of prespore cells. Our preliminary studies (V. M. Bowers \& K. L. Williams, unpublished) suggest that it is an adhesion molecule involved in heterophilic binding. To form a tissue, cells must adhere and here we claim that the PsA cell surface molecule, which is morphogen (cAMP)-regulated, is expressed in a spatial manner. It is not apparent in young slugs of strain WS380B, but coincides with the slug maturation which involves shortening by speeding up of the rearmost cells (Breen et al., 1992). We argue that the mature $D$. discoideum slug tissue has more adhesive prespore cells in the rear, which maintain the compact shape of the back of the mature slug.

The research was supported by NH and MRC Grant 890627 to K. L.W. and John Redmond. L.H.B. is a recipient of a Commonwealth Postgraduate Research Award.

\section{References}

BARKLIS, E. \& Lodish, H. F. (1983). Regulation of Dictyostelium discoideum mRNAs specific for prespore or prestalk cells. Cell 32, $1139-1148$.

Bernstein, R. L., Browne, L. H., Yu, S. C. \& Williams, K. L. (1988). Detergent treatment of Dictyostelium discoideum cells allows examination of internal cell type-specific antigens by flow cytometry. Cytometry 9, 68-74.

BoNNER, J. T. (1952). The pattern of differentiation in amoeboid slime molds. American Naturalist 86, 79-89. 
Breen, E. J., Eliott, S., Vardy, P. H., White, A. \& Williams, K. L. (1992). Length regulation in the Dictyostelium discoideum slug is a late event. Journal of Experimental Zoology 262, 299-306.

Browne, L. H., Sadeghi, H., Blumberg, D., Williams, K. L. \& KLEIN, C. (1989). Re-expression of 117 antigen, a cell surface glycoprotein of aggregating cells, during terminal differentiation of Dictyostelium discoideum prespore cells. Development 105, 657-664.

Early, A., McRobbie, S. J., Duffy, K. T., Jermyn, K. A., Tilly, R., Ceccarelli, A. \& Williams, J. G. (1988). Structural and functional characterization of genes encoding Dictyostelium prestalk and prespore cell-specific proteins. Developmental Genetics 9, 383-402.

FIRTEL, R. A. (1991). Signal transduction pathways controlling multicellular development in Dictyostelium. Trends in Genetics 7, 381-388.

Gomer, R. H., Datta, S. \& Firtel, R. A. (1986). Cellular and subcellular distribution of a cAMP-regulated prestalk protein and prespore protein in Dictyostelium discoideum. A study on the ontogeny of prestalk and prespore cells. Journal of Cell Biology 103, 1999-2015.

Gooley, A. A., Marshchalek, R. \& Williams, K. L. (1992). Size polymorphisms due to changes in the number of $O$-glycosylated tandem repeats in the Dictyostelium discoideum glycoprotein PsA. Genetics 130, 749-756.

GregG, J. H., Krefft, M., HaAs-Kraus, A. \& Williams, K. L. (1982). Antigenic differences detected between prespore cells of Dictyostelium discoideum and Dictyostelium mucoroides using monoclonal antibodies. Experimental Cell Research 142, 229-233.

Gross, J. D., Bradbury, J., Kay, R. R. \& Peacey, M. J. (1983). Intracellular $\mathrm{pH}$ and the control of cell differentiation in Dictyostelium discoideum. Nature, London 303, 244-245.

GuRDON, J. B. (1992). The generation of diversity and pattern in animal development. Cell 68, 185-199.

HABERSTROH, L. \& FIRTEL, R. A. (1990). A spatial gradient of expression of a cAMP-regulated prespore cell-type-specific gene in Dictyostelium. Genes and Development 4, 596-612.

Jermyn, K. A., Duffy, K. T. I. \& Williams, J. G. (1989). A new anatomy of the prestalk zone of Dictyostelium. Nature, London $\mathbf{3 4 0}$, 144-146.

Kay, R. R., GaRroD, D. \& Tilly, R. (1978). Requirements for cell differentiation in Dictyostelium discoideum. Nature, London 271, 58-60.

Krefft, M., Voet, L., GregG, J. H., Mairhofer, H. \& Williams, K. L. (1984). Evidence that positional information is used to establish the prestalk-prespore pattern in Dictyostelium discoideum aggregates. EMBO Journal 3, 201-206.

KrefFt, M., Voet, L., GregG, J. H. \& Williams, K. L. (1985). Use of a monoclonal antibody recognizing a cell surface determinant to distinguish prestalk and prespore cells of Dictyostelium discoideum slugs. Journal of Embryology and Experimental Morphology 88, $15-24$.

Kwong, L., Sobolewski, A., Atkinson, L. \& Weeks, G. (1988). Stalk cell formation in monolayers from isolated prestalk and prespore cells of Dictyostelium discoideum: evidence for two populations of prestalk cells. Development 104, 121-127.

Lam, T. Y., Pickering, G., Geltosky, J. \& SiU, C. H. (1981). Differential cell cohesiveness expressed by prespore and prestalk cells of Dictyostelium discoideum. Differentiation 20, 22-28.

MCAllister, L. \& Goodman, C. S. (1992). Dynamic expression of the cell adhesion molecule fasciclin I during embryonic development in Drosophila. Development 115, 267-276.

Morris, H. R., TaYlor, G. W., Masento, M. S., Jermyn, K. A. \& KAY, R. R. (1987). Chemical structure of the morphogen differentiation inducing factor from Dictyostelium discoideum. Nature, London 328, 811-814.
MoRrissey, J. H. (1982). Cell proportioning and pattern formation. In The Development of Dictyostelium discoideum, pp. 411-449. Edited by W. F. Loomis. London: Academic Press.

NoJi, S., Nohno, T., Koyama, E., Muto, K., Ohyama, K., Aoki, Y., Tamura, K., Ohsugi, K., Ide, H., Taniguchi, S. \& Saito, T. (1991). Retinoic acid induces polarizing activity but is unlikely to be a morphogen in the chick limb bud. Nature, London 350, 83-86.

NUSSLEIN-VOLHARD, C. (1991). Determination of the embryonic axes of Drosophila. Development 1991 Supplement 1, 1-10.

Odell, G. M. \& BONNER, J. T. (1986). How the Dictyostelium discoideum grex crawls. Philosophical Transactions of the Royal Society, London B312, 487-525.

Otte, A. P., Plomp, M. J. E., Arents, J. C., Janssens, P. M. W. \& Van DRIEL, R. (1986). Production and turnover of cAMP signals by prestalk and prespore cells in Dictyostelium discoideum cell aggregates. Differentiation 32, 185-191.

RAPER, K. B. (1984). The Dictyostelids. New Jersey: Princeton University Press.

St Johnston, D. \& Nusslein-Volmard, C. (1992). The origin of pattern and polarity in the Drosophila embryo. Cell 68, 201-219.

ScHAAP, P. (1986). Regulation of size and pattern in the cellular slime molds. Differentiation 33, 1-16.

SCHAAP, P. \& WANG, M. (1986). Interactions between adenosine and oscillatory cAMP signalling regulate size and pattern in Dictyostelium. Cell 45, 137-144.

STERnFeld, J. \& David, C. N. (1981). Cell sorting during pattern formation in Dictyostelium. Differentiation 20, 10-21.

STERNFELD, J. \& DAVID, C. N. (1982). Fate and regulation of anteriorlike cells in Dictyostelium slugs. Developmental Biology 93, 111-118.

TAKEUCHI, I. (1963). Immunochemical and immunohistochemical studies on the development of the cellular slime mold Dictyostelium mucoroides. Developmental Biology 8, 1-26.

TAKEUCHI, I., HAYASHI, M. \& TASAKa, M. (1977). Cell differentiation and pattern formation in Dictyostelium. In Development and Differentiation in the Cellular Slime Moulds, pp. 1-16. Edited by $\mathrm{P}$. Cappuccinelli and J.M. Ashworth. North Holland: Elsevier Biomedical Press.

TASaKa, M., Noce, T. \& TakeuchI, I. (1983). Prestalk and prespore differentiation in Dictyostelium as detected by cell type-specific monoclonal antibodies. Proceedings of the National Academy of Sciences of the United States of America 80, 5340-5344.

ThAller, C. \& EICHELE, G. (1987). Identification and spatial distribution of retinoids in the developing chick limb bud. Nature, London 327, 625-628.

Voet, L., KrefFt, M., Bruderlein, M. \& Williams, K. L. (1985). Flow cytometer study of anterior-like cells in Dictyostelium discoideum. Journal of Cell Science 75, 423-435.

Wanek, N., Gardiner, D. M., Muneoka, K. \& Bryant, S. V. (1991). Conversion by retinoic acid of anterior cells into ZPA cells in the chick wing bud. Nature, London 350, 81-83.

WANG, M., VAN DRIEl, R. \& SchaAP, P. (1988). Cyclic AMPphosphodiesterase induces dedifferentiation of prespore cells in Dictyostelium discoideum slugs: evidence that cyclic AMP is the morphogenetic signal for prespore differentiation. Development 103, 611-618.

WeiJer, C. J. \& Durston, A. J. (1985). Influence of cyclic AMP and hydrolysis products on cell type regulation in Dictyostelium discoideum. Journal of Embryology and Experimental Morphology 86, 19-37.

WEST, C. M. \& MCMAHON, D. (1979). The axial distribution of plasma membrane molecules in pseudoplasmodia of the cellular slime mold Dictyostelium discoideum. Experimental Cell Research 124, 393-401.

Williams, J. G. (1988). The role of diffusible molecules in regulating the cellular differentiation of Dictyostelium discoideum. Development 103, 1-16. 